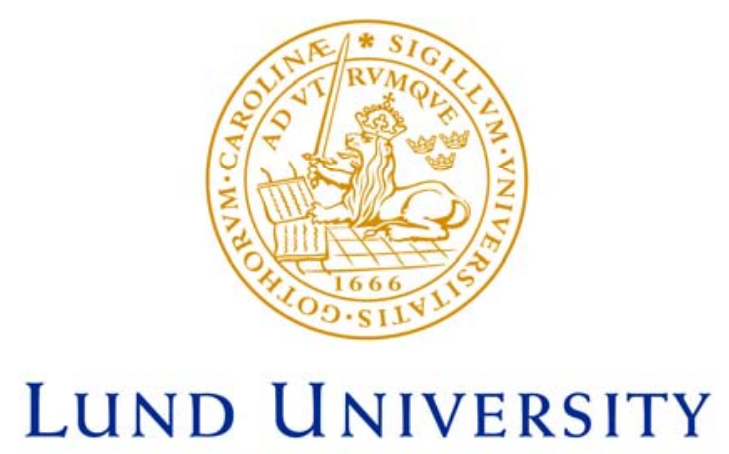

Faculty of Medicine

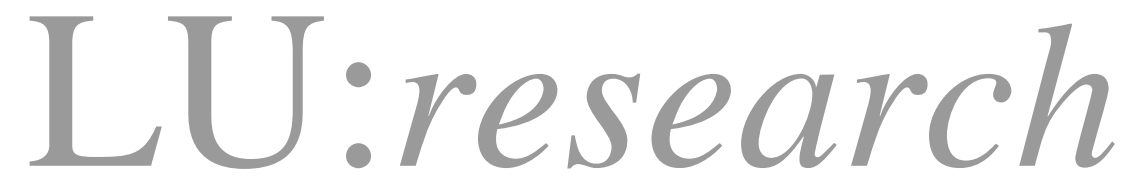

Institutional Repository of Lund University

This is an author produced version of a paper published in Inflammation research: official journal of the European Histamine Research Society. This paper has been peerreviewed but does not include the final publisher proofcorrections or journal pagination.

Citation for the published paper:

Mangell, $\mathrm{P}$ and Mihaesc $\mathrm{C}, \mathrm{A}$ and Wang, $\mathrm{Y}$ and Schramm,

$\mathrm{R}$ and Jeppsson, $\mathrm{B}$ and Thorlacius, $\mathrm{H}$.

"Critical role of P-selectin-dependent leukocyte recruitment in endotoxin-induced intestinal barrier dysfunction in mice."

Inflamm Res, 2007, Vol: 56, Issue: 5, pp. 189-94.

http://dx.doi.org/10.1007/s00011-007-6163-x

Access to the published version may

require journal subscription.

Published with permission from: Springer 


\section{Critical role of P-selectin-dependent leukocyte recruitment in endotoxin-induced intestinal barrier dysfunction in mice}

P. Mangell, A. Mihaescu, Y. Wang, R. Schramm, B. Jeppsson and H. Thorlacius

Department of Surgery, Malmö University Hospital, Lund University, Malmö, Sweden

\section{Correspondence to:}

Henrik Thorlacius

Department of Surgery

Malmö University Hospital

Lund University

SE-205 02 Malmö

Sweden

Phone: +46-40-331000

Telefax: +46-40-336207

henrik.thorlacius@med.lu.se

Keywords: sepsis, leukocyte, endotoxin, intestinal barrier, P-selectin

Running head: Leukocyte-recruitment in intestinal leakage. 


\begin{abstract}
Objective: To define the importance of leukocyte recruitment in endotoxin-induced gut permeability.
\end{abstract}

Materials and methods: 31 male C57BL/6 mice were challenged with lipopolysaccharide (LPS). Ileal permeability was measured in Ussing chambers and leukocyte-endothelium interactions studied with intravital fluorescence microscopy after $18 \mathrm{~h}$.

Results: LPS caused a clear-cut increase in leukocyte accumulation and intestinal permeability. Immunoneutralisation of P-selectin not only reduced leukocyte recruitment significantly (54\% reduction) but also abolished endotoxin-induced intestinal leakage. Intestinal levels of pro-inflammatory chemokines increased markedly in response to LPS but were not influenced by inhibition of P-selectin in vivo.

Conclusion: The present study shows not only that endotoxin-induced leukocyte recruitment is mediated by P-selectin but also that sepsis-associated intestinal leakage in the gut is largely regulated by leukocyte accumulation. Thus, our novel data demonstrate a critical link between P-selectin-dependent leukocyte recruitment and gut barrier failure in endotoxemia. 


\section{Introduction}

Despite substantial efforts to improve surgical treatment, antimicrobial therapies and immunomodulatory regimes, gram-negative sepsis remains a common cause of mortality in intensive care patients [1, 2]. Lipopolysaccharide (LPS) constitutes the major portion of the outer membrane of most clinically relevant gram-negative bacteria found in human infections [3]. The host response to LPS challenge is associated with disturbed gut integrity and increased leukocyte infiltration [4]. Indeed, increased intestinal leakage through the epithelial cell lining constitutes a key feature in the pathophysiology of sepsis by facilitating bacterial translocation and passage of toxic substances from the gastrointestinal tract [5]. Notably, several studies have reported that pro-inflammatory substances can increase the permeability of epithelial cell monolayers in vitro [6, 7]. However, these in vitro systems lack the presence of leukocytes and the potential role of leukocyte recruitment in mediating endotoxin-induced intestinal leakage in vivo remains therefore elusive.

LPS binds to the cell surface receptor CD14 on tissue macrophages and activates Toll-like receptor-4 [8], which, in turn, initiates intracellular signaling cascades that converge on specific transcription factors regulating gene expression of pro-inflammatory mediators, such as cytokines and chemokines [9]. Indeed, tissue recruitment of leukocytes is dependent on the formation and action of CXC chemokines, including macrophage inflammatory protein-2 (MIP-2) and cytokine-induced neutrophil chemoattractant (KC) [10]. Several studies have shown that leukocyte accumulation is a multistep process, comprising initial rolling along the microvascular endothelium followed by firm leukocyte adhesion and migration [11]. Leukocyte rolling is considered to be a precondition for the subsequent adhesion and extravasation of leukocytes and is largely dependent on the 
function of the selectin family of adhesion molecules (L-, E-, and P-selectin) [12, 13]. An accumulating body of data indicates that P-selectin supports most rolling adhesive interactions in vivo [14] although the detailed role of P-selectin in endotoxin-induced leukocyte-endothelium interactions in the small intestine has not previously been studied.

Based on the above considerations, the main aim of this study was to define the importance of leukocyte recruitment in endotoxemia-associated gut leakage. For this purpose, we used a monoclonal antibody against P-selectin, which we found to be effective in blocking LPSprovoked leukocyte-endothelium interactions in the intestine. 


\section{Methods}

\section{Animals}

Male C57BL/6 mice (Taconic Europa, Ry, Denmark) weighing between 20-22 g were maintained at $12 \mathrm{~h}$ dark and $12 \mathrm{~h}$ light cycles and had free access to standard pellet food (R3, Lactamin AB, Kimstad, Sweden) and water ad libitum. Anaesthesia was achieved by intraperitoneal (i.p.) injection of 7.5 mg ketamine hydrochloride (Hoffman-La Roche, Basel Switzerland) and 2.5 mg xylazine (Jansen Pharmaceutica, Beerse, Belgium) per 100 g body weight. All experiments were approved by the local Animal Ethic's Committee at Lund University.

\section{Experimental protocol}

Mice were allocated to one of the following groups: 1 ) negative control group received 0.3 ml sterile saline i.p. ; 2) positive control group received 2 mg lipopolysaccharide (LPS) from Escherichia coli serotype O111:B4 (Sigma Chemical Co, St Louis, MO, USA) per 100 g body weight, dissolved in $0.3 \mathrm{ml}$ sterile saline i.p. ; 3) control antibody group received $40 \mu \mathrm{g}$ of an isotype-matched rat antibody IgG (clone R3-34, BD Biosciences Pharmingen, San Diego, CA, USA) dissolved in sterile saline to a total volume of $0.2 \mathrm{ml}$ intravenously (i.v.) by a lateral tail vein injection, immediately followed by i.p. administration of LPS as described above; 4) anti-P-selectin antibody group received $40 \mu \mathrm{g}$ of a monoclonal antibody against mouse P-selectin (clone RB40.34, BD Biosciences Pharmingen) dissolved in sterile saline to a total volume of $0.2 \mathrm{ml}$ i.v. immediately followed by LPS as described above. Permeability studies and intravital microscopy were conducted $18 \mathrm{~h}$ after LPS treatment. 


\section{Intestinal permeability studies}

Mice in the separate groups were anaesthetised and a midline laparotomy was performed. The ileocaecal junction was identified and 3-4 cm of distal ileum was harvested while carefully removing the mesentery. The intestine was cut open along the mesenteric side, rinsed in Krebs' buffer and mounted in a modified Ussing diffusion chamber [15] (Harvard Apparatus, Holliston, MA, USA) using inserts allowing for a $0.25 \mathrm{~cm}^{2}$ exposed area of intestine. The chambers were filled with 3 ml Krebs' buffer that was continuously bubbled with carbogen $\left(95 \% \mathrm{O}_{2}\right.$ and $\left.5 \% \mathrm{CO}_{2}\right)$ at $37^{\circ} \mathrm{C}$. The experiment started within 45 min after harvesting the intestinal segment by first replacing the buffer in the serosal (recipient) reservoir with $3 \mathrm{ml}$ fresh Krebs' buffer and then the buffer in the mucosal (donor) reservoir with 3 ml Krebs' buffer containing sodium fluorescein (molecular weight 376 Da, Sigma Chemical Co, St Louis, MO, USA) at a concentration of $0.1 \mathrm{mg} / \mathrm{ml}$. The chambers were covered to prevent light exposure. After 60 min specimens were taken from the serosal chambers for spectrofluorometry (SpectaMax Gemini, Molecular Devices, Sunnyvale, CA, USA). Samples were serially diluted in duplicates and measured at an excitation wavelength of $485 \mathrm{~nm}$ and emission wavelength of $525 \mathrm{~nm}$. Known amounts of sodium fluorescein were dissolved in Krebs' buffer to make a standard curve, which was used to determine the amount of sodium fluorescein passage in the experiments.

The transepithelial potential differences were measured immediately before start of the experiment $(\mathrm{t}=0)$ and after $60 \mathrm{~min}$, using electrodes imbedded in $3 \mathrm{M} \mathrm{KCl}$ agar connected to a standard commercial voltmeter (APPA 63N Multimeter, APPA Technology Corp, Taipai, Taiwan). Potential difference $<3.0 \mathrm{mV}$ at $\mathrm{t}=0$ excluded a specimen from the experiment. 


\section{Intravital microscopy}

Mice were anaesthetised and placed on a heating pad $\left(37^{\circ} \mathrm{C}\right)$ to maintain body temperature. A polyethylene catheter (PE-10) with an inner diameter of $0.28 \mathrm{~mm}$ was inserted into the jugular vein and used to administer marker solutions and additional anaesthesia. A midline laparotomy was performed and the most distal part of ileum was carefully exteriorised. The mouse was then put under an inverted Olympus microscope (IX70, Olympus Optical Co, GmgH, Hamburg, Germany), equipped with different lenses (x10/NA 0.25 and x40/NA 0.60). The image was televised using a charge-coupled device video camera (FK 6990A-IQ, Pieper GmbH, Schwerte, Germany) and recorded on videotape (Panasonic HR S8600 S-VHS recorder) for later off-line evaluation. Analysis of rolling and adhesion was made in $3-6$ distal ileum venules with an inner diameter of 25 $50 \mu \mathrm{m}$ and with stable blood flow. Blood perfusion was studied after contrast enhancement of the plasma with i.v. injection of $0.1 \mathrm{ml}$ fluorescein isothiocyanate-dextran (molecular weight 150 000, 5 mg/ml, Sigma Chemical Co, St Louis, MO, USA) followed by illumination with blue light (excitation wavelength $490 \mathrm{~nm}$, emission wavelength $510 \mathrm{~nm}$ ).

In vivo labelling of leukocytes was done by i.v. injection of $0.1 \mathrm{ml}$ rhodamine $6 \mathrm{G}$ (molecular weight 479, 0.5 mg/ml, Sigma Chemical Co, St Louis, MO, USA) followed by illumination with green fluorescent light (excitation wavelength $530 \mathrm{~nm}$, emission wavelength $560 \mathrm{~nm}$ ). Leukocyte rolling was determined by counting the number of leukocytes passing a reference point in the venule during a 20 sec observation period and expressed as cells/min. Firm adhesion was determined by counting the number of leukocytes adhering and remaining stationary along a $100 \mu \mathrm{m}$ segment of the venular endothelium for $20 \mathrm{sec}$. The results are expressed as cells/mm venule length. Blood flow velocity was analysed off-line by means of a computer image analysis programme 
(CapImage, Zeintl, Heidelberg, Germany). The velocity is expressed as mm/sec and wall shear rate calculated from the formula wall shear rate $=8([$ velocity/1.6]/venular diameter $)$ [16].

\section{ELISA}

The levels of chemokines MIP-2 and KC were determined in distal ileum tissue. After intravital microscopy a segment of the distal ileum was harvested, rinsed of faecal matter, weighed and put in PBS containing 1\% PEST (penicillin and streptomycin) (Gibco/Invitrogen, Carlsbad, CA, USA) and $0.1 \mathrm{mg} / \mathrm{ml}$ amphotericin B (Fungizone, Bristol Myers Squibb, NY, NY, USA) for 60 min. The tissue was then incubated in Dulbecco's modified Eagle’s medium solution containing 10\% fetal calf serum, 1\% PEST and amphotericin B for $24 \mathrm{~h}$ in $37^{\circ} \mathrm{C}$ in a 12-well plate. The medium was harvested, centrifuged for 10 min at $3000 \mathrm{rpm}$ and the supernatant was then collected and frozen in $-20^{\circ} \mathrm{C}$. Analysis was made using a quantitative sandwich enzyme immunoassay technique with polyclonal antibodies specific for murine MIP-2 and KC (R\&D Systems, Minneapolis, MN, USA). The minimum detectable amount of protein in these kits is 1.5 $\mathrm{pg} / \mathrm{ml}$. Each sample was analysed in duplicates and optical density read at $450 \mathrm{~nm}$ (Milenia Kinetic Analyzer, DPC, Los Angeles, CA, USA).

\section{Myeloperoxidase (MPO)}

Two cm of distal ileum were harvested and weighed for MPO assay from the negative control group ( $n=4)$, control antibody group $(n=8)$ and anti-P-selectin antibody group $(\mathrm{n}=5)$. After homogenisation in $1.5 \mathrm{ml} \mathrm{0.5 \%}$ hexadecyltrimethylammonium bromide (Sigma Chemical, St Louis, MO, USA) MPO was extracted and purified. Absorbance was 
measured in a spectrophotometer at $450 \mathrm{~nm}$ and the MPO activity calculated from a standard curve and corrected for sample weight.

\section{Systemic leukocyte counts}

$20 \mu \mathrm{l}$ peripheral blood was mixed with Turks solution $(0.2 \mathrm{mg}$ gentian violet/ml glacial acetic acid, $6.25 \% \mathrm{v} / \mathrm{v}$ ) in a 1:10 dilution. Using a Burker chamber, leukocytes were counted and differentiated as mononuclear (MNL) or polymorphonuclear (PMNL).

\section{Statistical analysis}

Data are presented as mean values \pm SEM. Statistical evaluations were performed using one way analysis of variance (ANOVA) followed by multiple comparisons between groups (Holm-Sidak's method). For non-parametric data Kruskal-Wallis one way analysis of variance on ranks followed by multiple comparison between groups (Dunn's method) was used. $P<0.05$ was considered significant and $n$ represents the number of animals. 


\section{Results}

\section{Endotoxin-induced intestinal permeability}

Challenge with LPS markedly increased sodium fluorescein permeability by $104 \%$ in the distal ileum. Thus, after 60 min in the Ussing-chamber, the concentration of sodium fluorescein was $0.22 \pm 0.01 \mu \mathrm{g} / \mathrm{ml}$ on the serosal side of distal ileum in LPS-treated mice ( $n=11)$, as compared to $0.11 \pm 0.01 \mu \mathrm{g} / \mathrm{ml}$ in negative control mice $(n=8, P<0.05)$ (Fig. 1). In control antibody-treated mice, LPS significantly increased intestinal permeability by $68 \%(0.19 \pm 0.01 \mu \mathrm{g} / \mathrm{ml}, n=6, P<0.05$ vs negative control). Notably, pre-treatment with the anti-P-selectin antibody completely abolished LPS-induced permeability, normalising passage of sodium fluorescein across distal ileum down to control levels $(0.12 \pm 0.01$ $\mu \mathrm{g} / \mathrm{ml}, n=6, P<0.05$ vs LPS). Electrical potential difference across the intestinal membrane remained constant and was not affected by LPS or any of the antibodies $(n=6-$ 11) (Table 1).

\section{Endotoxin-induced leukocyte recruitment}

Intravital microscopy revealed that baseline leukocyte rolling was $12 \pm 1$ cells/min in negative control mice. Concomitant with increased permeability, it was observed that LPS significantly increased leukocyte rolling up to $30 \pm 6$ cells $/ \min (n=6, P<0.05$ vs negative control) (Fig. 2). In contrast, we found that immunoneutralisation of P-selectin decreased leukocyte rolling down to baseline levels, i.e. $6 \pm 2$ cells $/ \mathrm{min}(n=6, P<0.05$ vs control antibody + LPS) (Fig. 2).

The number of leukocytes firmly adhering to the venular wall was $1.0 \pm 0.4$ cells $/ \mathrm{mm}$ in the negative control group and increased significantly after LPS-challenge up to $48 \pm 9$ 
cells/mm ( $n=6, P<0.05$ vs negative control) (Fig. 3). Administration of the control antibody had no effect on leukocyte adhesion in endotoxemic mice (48 \pm 5 cells $/ \mathrm{mm}$ ). However, pre-treatment with the anti-P-selectin antibody reduced the number of adherent leukocytes down to $22 \pm 5$ cells/min, corresponding to 54\% reduction in LPS-induced leukocyte adhesion ( $\mathrm{n}=6, \mathrm{P}<0.05 v s$ control antibody + LPS).

There were no significant differences in vessel diameter or blood flow velocity between the different experimental groups (Table 2). Moreover, differential count of the peripheral leukocytes revealed a 96\% reduction of MNL in the LPS-treated mice (Table 3). This reduction was absent in the endotoxemic animals pre-treated with the anti-P-selectin antibody. No significant changes in PMNL were observed between the groups.

Moreover, tissue infiltration of leukocytes was evaluated by measuring intestinal levels of MPO. We found that LPS increased MPO activity in the intestine by more than two-fold (n $=4-8, P<0.05 v s$ negative control). Notably, it was observed that pre-treatment with the anti-P-selectin antibody reduced MPO levels from $0.7 \pm 0.1$ units/mg down to $0.4 \pm 0.1$ units/mg (n = 5-8, $P<0.05$ vs control antibody + LPS).

\section{Endotoxin-induced chemokine production}

LPS challenge significantly increased protein levels of CXC chemokines in the intestine (Fig. $4 \mathrm{a}$ and b). Thus, baseline values of MIP-2 and KC in the small intestine were $35.5 \pm$ $10.6 \mathrm{pg} / \mathrm{mg}$ and $66.5 \pm 18.7 \mathrm{pg} / \mathrm{mg}$, respectively. LPS resulted in more than a three-fold increase in tissue chemokines, i.e. MIP-2 increased up to $154.4 \pm 35.0(n=7, P<0.05$ vs negative control) (Fig. 4 a) and $\mathrm{KC}$ up to $177.0 \pm 28.6$ ( $n=7, P<0.05$ vs negative control) (Fig. 4 b). Interestingly, immunoneutralisation of P-selectin had no effect on the levels of 
MIP-2 or KC in intestine of endotoxemic mice. No differences in tissue chemokines were found between the control antibody group and the anti-P-selectin group. 


\section{Discussion}

This study demonstrates a fundamental role of leukocytes in mediating sepsis-associated intestinal barrier dysfunction. Indeed, inhibition of P-selectin not only blocked endotoxininduced leukocyte rolling and adhesion, but also abolished the increased intestinal permeability in endotoxemic animals. These novel findings suggest a causal link between on one hand leukocyte accumulation and on the other hand intestinal leakage in sepsis. Moreover, it was found that inhibition of P-selectin did not exert any effect on local production of pro-inflammatory mediators in the intestine, suggesting that actual infiltration of leukocytes is relatively more important than any direct effect of proinflammatory mediators on tissue cells during induction of intestinal permeability in sepsis. Thus, our data indicate that targeting P-selectin and leukocyte-endothelial interactions may be a useful approach to attenuate sepsis-induced intestinal leakage.

An intact mucosal barrier function maintained by the intestinal epithelial cells is crucial in preventing toxic substances in the gut lumen from entering the body. The integrity of the epithelial cell lining in the gut mucosa is also critical in preventing the huge load of potentially pathogenic bacteria contained within the lumen to translocate into normally sterile compartments of the body. These protective functions of the intestinal mucosa are delicately balanced with the task of absorbing water, ions and nutrients from the intestinal lumen and also allowing small quantities of antigens to permeate and interact with the gut immune system [17]. In sepsis this balance is disturbed, resulting in an increased permeability. Indeed, sepsis is also associated with leukocyte activation and recruitment. However, the relation between leukocyte-endothelial interactions and increased permeability across the intestinal wall is not known. The present study is the first one to explore the definitive role of leukocytes in gut barrier dysfunction associated with a 
systemic inflammatory response in vivo. We used a model based on systemic administration of LPS, which increased leakage in the gut by more than $100 \%$. Notably, using this model, we found that immunoneutralisation of P-selectin not only abolished intestinal recruitment of leukocytes but also markedly decreased endotoxin-induced permeability in the distal small intestine. In this context it is important to note that Pselectin expression is limited to endothelial cells and circulating platelets [18] and is neither expressed in the extravascular matrix nor on tissue cells in the intestinal wall, excluding the possibility of any effect of the anti-P-selectin antibody on targets beyond the intestinal microvasculature. MPO activity is a useful indicator of leukocyte recruitment, and our results shows that LPS treatment induces a significant increase in leukocyte recruitment in the intestine. This effect was markedly reduced by inhibition of P-selectin, which is in line with our findings on leukocyte rolling and adhesion. Taken together, these novel findings suggest that leukocyte recruitment plays a critical role in mediating intestinal barrier dysfunction induced by endotoxin.

Moreover, it is interesting to note that inhibition of P-selectin reduced leukocyte recruitment and intestinal leakage, but had no concomitant effect on tissue production of pro-inflammatory mediators (MIP-2 and KC) in the intestinal wall. The fact that the intestinal levels of MIP-2 and KC were intact in anti-P-selectin antibody-treated animals suggests that leukocyte recruitment is relatively more important than the local production of pro-inflammatory mediators in the pathophysiology of sepsis-associated gut barrier dysfunction. This notion is somewhat in contrast to previous in vitro studies reporting that pro-inflammatory mediators exert powerful effects on the permeability across epithelial cell monolayers [6, 7]. However, it is of importance to note that these in vitro studies lack the presence of leukocytes, which we, herein, have shown to play a key role in sepsis- 
associated intestinal leakage in vivo. Although the predominant impact on the intestinal tissue seems to be regulated by accumulated leukocytes, our data do not exclude that local mediators also may contribute to increased leakage by exerting direct and leukocyteindependent effects in the intestinal tissue.

The underlying mechanism behind the leukocyte-mediated intestinal barrier dysfunction observed herein remains elusive. Indeed, leukocytes contain numerous potent and tissue damaging substances, such as reactive oxygen species, bactericidal permeability-increasing proteins, matrix metalloproteinases, defensins and elastases [19]. Upon release in the intestinal wall these may contribute to disrupted intestinal integrity and increased macromolecular passage across the epithelial cell lining. In this study, we used sodium fluorescein as a marker of intestinal permeability. It is generally held that sodium fluorescein permeates paracellularly across the intestinal epithelium [20]. Paracellular integrity is maintained by tight junctions, which consists mainly of different transmembrane proteins [21]. Interestingly, it has been shown that neutrophil-derived proteases have the capacity to disrupt the function of tight junctions [22] which may help to explain, at least in part, the underlying mechanism of leukocyte-dependent gut barrier dysfunction in sepsis. Besides, it may be worth noting that leukocyte migration per se may also cause physical disruption of the mucosal integrity and thereby increase intestinal leakage [23].

When inducing permeability changes in the gastrointestinal tract by means of exogenous administration of LPS, it is of importance to determine if the increased permeability is due to any effect on the viability of the enterocytes. For this purpose we measured the electrical potential difference, which is maintained by ATP-dependent ion pumps in the basolateral 
membrane of intact enterocytes. Throughout the 60 min of this experiment, we did not find any significant difference in potential difference between LPS-treated and control mice when the intestinal segments were mounted in the Ussing chambers, indicating that LPS did not have any adverse effect on tissue viability. This notion is in accordance with the findings of Wells et al reporting that LPS has no effect on enterocyte morphology or viability [24].

In conclusion, this study demonstrates for the first time that leukocyte recruitment per se is critical in mediating sepsis-associated intestinal barrier dysfunction. Interestingly, the accumulation of leukocytes seems to be more important than pro-inflammatory mediators in regulating LPS-induced intestinal leakage. Our data show that targeting P-selectin function decreases pathological leakage in the gut through inhibition of leukocyte accumulation. Thus, these novel findings suggest that therapies directed against recruitment of leukocytes may be a useful strategy against gut barrier failure in sepsis. 


\section{Acknowledgements:}

The authors wish to thank Susanne Eiswohld for excellent technical assistance. This study was supported by grants from Swedish Medical Research Council (2002-995, 2002-8012, 2003-4661, Crafoordska Foundation, Blanceflor's Foundation, Einar and Inga Nilsson’s Foundation, Harald and Greta Jaensson’s Foundation, Greta and Johan Kock's Foundation, Fröken Agnes Nilsson’s Foundation, Magnus Bergvall’s Foundation, Mossfelt's Foundation, Nanna Svartz' Foundation, Ruth and Richard Julin's Foundation, Swedish Medical Assocation, Tegger’s Foundation, Dir. A. Påhlsson’s Foundation, Gunnar Nilsson's Foundation, Apotekare Hedberg's Foundation and Bengt Ihre's Foundation. 


\section{Reference list}

[1] Angus DC, Linde-Zwirble WT, Lidicker J, Clermont G, Carcillo J, Pinsky MR. Epidemiology of severe sepsis in the United States: analysis of incidence, outcome, and associated costs of care. Crit Care Med 2001;29:1303-1310.

[2] Angus DC, Wax RS. Epidemiology of sepsis: an update. Crit Care Med 2001;29:S109-S116.

[3] Opal SM, Cohen J. Clinical gram-positive sepsis: does it fundamentally differ from gram-negative bacterial sepsis? Crit Care Med 1999;27:1608-1616.

[4] Garcia SF, Liaudet L, Marton A, Hasko G, Batista LC, Deitch EA et al. Inosine improves gut permeability and vascular reactivity in endotoxic shock. Crit Care Med 2001;29:703-708.

[5] De Souza DA, Greene LJ. Intestinal permeability and systemic infections in critically ill patients: effect of glutamine. Crit Care Med 2005;33:1125-1135.

[6] Diebel LN, Liberati DM, Baylor AE, III, Brown WJ, Diglio CA. The pivotal role of tumor necrosis factor-alpha in signaling apoptosis in intestinal epithelial cells under shock conditions. J Trauma 2005;58:995-1001.

[7] Schmitz H, Fromm M, Bentzel CJ, Scholz P, Detjen K, Mankertz J et al. Tumor necrosis factor-alpha (TNFalpha) regulates the epithelial barrier in the human intestinal cell line HT-29/B6. J Cell Sci 1999;112 ( Pt 1):137-146.

[8] Chow JC, Young DW, Golenbock DT, Christ WJ, Gusovsky F. Toll-like receptor-4 mediates lipopolysaccharide-induced signal transduction. J Biol Chem 1999;274:10689-10692. 
[9] Palsson-McDermott EM, O'Neill LA. Signal transduction by the lipopolysaccharide receptor, Toll-like receptor-4. Immunology 2004;113:153-162.

[10] Li X, Klintman D, Liu Q, Sato T, Jeppsson B, Thorlacius H. Critical role of CXC chemokines in endotoxemic liver injury in mice. J Leukoc Biol 2004;75:443-452.

[11] Butcher EC. Leukocyte-endothelial cell recognition: three (or more) steps to specificity and diversity. Cell 1991;67:1033-1036.

[12] Carlos TM, Harlan JM. Leukocyte-endothelial adhesion molecules. Blood 1994;84:2068-2101.

[13] Thorlacius H. Selectins as targets of inflammatory diseases. Curr Med Chem 2004;3:31-38.

[14] Klintman D, Li X, Thorlacius H. Important Role of P-Selectin for Leukocyte Recruitment, Hepatocellular Injury, and Apoptosis in Endotoxemic Mice. Clin Diagn Lab Immunol 2004;11:56-62.

[15] Grass GM, Sweetana SA. In vitro measurement of gastrointestinal tissue permeability using a new diffusion system. Pharm Res 1988;5:372-376.

[16] House SD, Lipowsky HH. Leukocyte-endothelium adhesion: Microhemodynamics in mesentery of the cat. Microvascular Research 1987;34:363-379.

[17] Acheson DW, Luccioli S. Microbial-gut interactions in health and disease. Mucosal immune responses. Best Pract Res Clin Gastroenterol 2004;18:387-404.

[18] McEver RP, Beckstead JH, Moore KL, Marshall-Carlson L, Bainton DF. GMP-140, a platelet alpha-granule membrane protein, is also synthesized by vascular 
endothelial cells and is localized in Weibel-Palade bodies. J Clin Invest 1989;84:9299.

[19] Faurschou M, Borregaard N. Neutrophil granules and secretory vesicles in inflammation. Microbes Infect 2003;5:1317-1327.

[20] Bernkop-Schnurch A, Clausen AE, Guggi D. The use of auxiliary agents to improve the mucosal uptake of peptides. Medicinal Chemistry Reviews 2004;1:1-10.

[21] Balda MS, Matter K. Transmembrane proteins of tight junctions. Seminars in Cell \& Developmental Biology 2000;11:281-289.

[22] Ginzberg HH, Cherapanov V, Dong Q, Cantin A, McCulloch CA, Shannon PT et al. Neutrophil-mediated epithelial injury during transmigration: role of elastase. Am J Physiol Gastrointest Liver Physiol 2001;281:G705-G717.

[23] Nash S, Stafford J, Madara JL. Effects of polymorphonuclear leukocyte transmigration on the barrier function of cultured intestinal epithelial monolayers. J Clin Invest 1987;80:1104-1113.

[24] Wells CL, Jechorek RP, Olmsted SB, Erlandsen SL. Effect of LPS on epithelial integrity and bacterial uptake in the polarized human enterocyte-like cell line Caco-2. Circulatory Shock 1993;40:276-288. 


\section{Figure Legends}

\section{Figure 1}

Mucosal-to-serosal permeability of sodium fluorescein $(\mu \mathrm{g} / \mathrm{ml})$ in distal ileum. Mice were pre-treated with PBS, control-antibody (control ab) or anti-P-selectin antibody (anti-Pselectin $\mathrm{ab}$ ) and then challenged with LPS ( $2 \mathrm{mg} / 100 \mathrm{~g}$ body weight) for 18 hours. Values are mean \pm SEM. $\# P<0.05$ vs negative control and $* P<0.05$ vs positive control (LPS) or control-antibody + LPS.

\section{Figure 2}

Leukocyte rolling (cells/min) along the endothelium of postcapillary venules in the submucosa of distal ileum was measured by use of intravital microscopy. Mice were pretreated with PBS, control-antibody (control ab) or anti-P-selectin antibody (anti-P-selectin ab) and then challenged with LPS (2 mg/100 g body weight) for 18 hours.

Values are mean \pm SEM. $\# P<0.05$ vs negative control and $* P<0.05$ vs positive control (LPS) or control-antibody + LPS.

\section{Figure 3}

Leukocyte adhesion (cells/mm) on the endothelium of postcapillary venules in the submucosa of distal ileum was measured by use of intravital microscopy. Mice were pretreated with PBS, control-antibody (control ab) or anti-P-selectin antibody (anti-P-selectin ab) and then challenged with LPS (2 mg/100 g body weight) for 18 hours.

Values are mean \pm SEM. $\# P<0.05$ vs negative control and $* P<0.05$ vs negative control, positive control (LPS) or control-antibody + LPS.

\section{Figure 4}


Levels of the a) macrophage inflammatory protein-2 (MIP-2) and b) cytokine-induced neutrophil chemoattractant (KC) in distal ileum were measured by ELISA. Mice were pretreated with PBS, control-antibody (control ab) or anti-P-selectin antibody (anti-P-selectin $\mathrm{ab}$ ) and then challenged with LPS (2 mg/100 g body weight) for 18 hours.

Values are mean \pm SEM and $\# P<0.05$ vs negative control. 


\section{Table 1. Potential difference}

\begin{tabular}{lccc}
\hline & $n$ & $\mathrm{t}=0$ & $\mathrm{t}=60$ \\
\hline Negative control & 8 & $7.2 \pm 0.5$ & $5.8 \pm 0.7$ \\
PBS + LPS & 11 & $7.5 \pm 0.4$ & $4.7 \pm 0.5$ \\
Control ab + LPS & 6 & $5.9 \pm 0.7$ & $4.9 \pm 0.9$ \\
Anti-P-selectin ab + LPS & 6 & $6.2 \pm 1.6$ & $4.6 \pm 0.7$ \\
\hline
\end{tabular}

Potential difference (mV) across intestinal segments mounted in Ussing chambers. Mice were pretreated with PBS, control-antibody (control ab) or anti-P-selectin antibody (anti-P-selectin ab) and then challenged with LPS ( $2 \mathrm{mg} / 100 \mathrm{~g}$ body weight) for 18 hours. $\mathrm{t}=0$ indicates the time point immediately prior to addition of sodium fluorescein on the mucosal side of the intestinal segments and $t=60$ at the end of the experiment. Values are mean \pm SEM. 
Table 2. Haemodynamic parameters.

\begin{tabular}{lcccc}
\hline & $n$ & Diameter $(\mu \mathrm{m})$ & Velocity $(\mathrm{mm} / \mathrm{sec})$ & Wall shear rate $\left(\mathrm{sec}^{-1}\right)$ \\
\hline Negative control & 6 & $29.4 \pm 2.9$ & $1.0 \pm 0.1$ & $142 \pm 23$ \\
PBS + LPS & 6 & $37.7 \pm 3.8$ & $0.8 \pm 0.1$ & $105 \pm 24$ \\
Control ab + LPS & 6 & $35.1 \pm 2.0$ & $0.8 \pm 0.1$ & $95 \pm 13$ \\
Anti-P-selectin ab + LPS & 6 & $32.3 \pm 2.5$ & $0.7 \pm 0.1$ & $102 \pm 15$ \\
\hline
\end{tabular}

Haemodynamic parameters of distal ileum venules. Mice were pre-treated with PBS, control-

antibody (control ab) or anti-P-selectin antibody (anti-P-selectin ab) and then challenged with LPS ( $2 \mathrm{mg} / 100 \mathrm{~g}$ body weight) for 18 hours. Values are mean \pm SEM. Wall shear rate $=$

8([velocity/1.6]/venular diameter. 
Table 3. Peripheral leukocyte counts.

\begin{tabular}{lccc}
\hline & $n$ & $\begin{array}{c}\text { Mononuclear } \\
\text { leukocytes } \\
\left(10^{6} / \mathrm{ml}\right)\end{array}$ & $\begin{array}{c}\text { Polymorphonuclear } \\
\text { leukocytes } \\
\left(10^{6} / \mathrm{ml}\right)\end{array}$ \\
\hline Negative control & 6 & $2.3 \pm 0.1$ & $0.6 \pm 0.3$ \\
PBS + LPS & 6 & $0.1 \pm 0.01^{*}$ & $0.2 \pm 0.05$ \\
Control ab + LPS & 6 & $0.1 \pm 0.01^{*}$ & $0.1 \pm 0.04$ \\
Anti-P-selectin ab + LPS & 6 & $0.6 \pm 0.2$ & $0.5 \pm 0.2$ \\
\hline
\end{tabular}

Peripheral leukocyte counts. Values are mean \pm SEM. ${ }^{*} P<0.05$ vs negative control. 


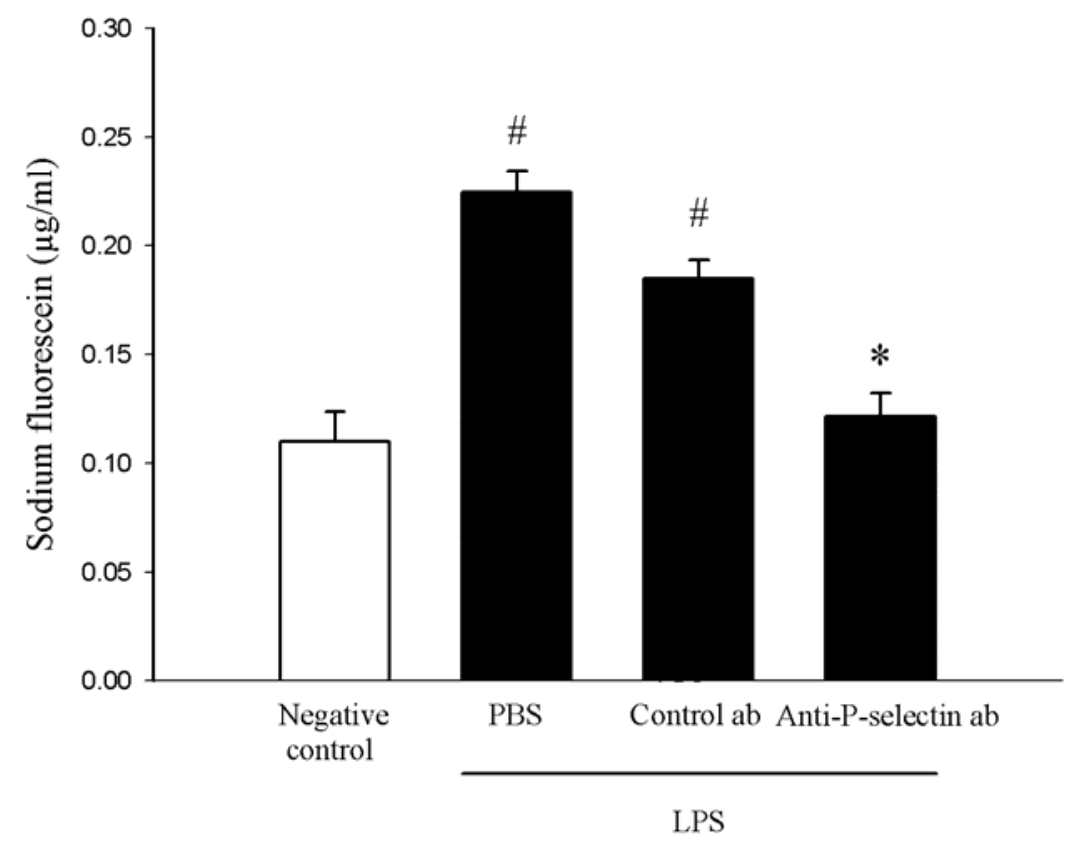




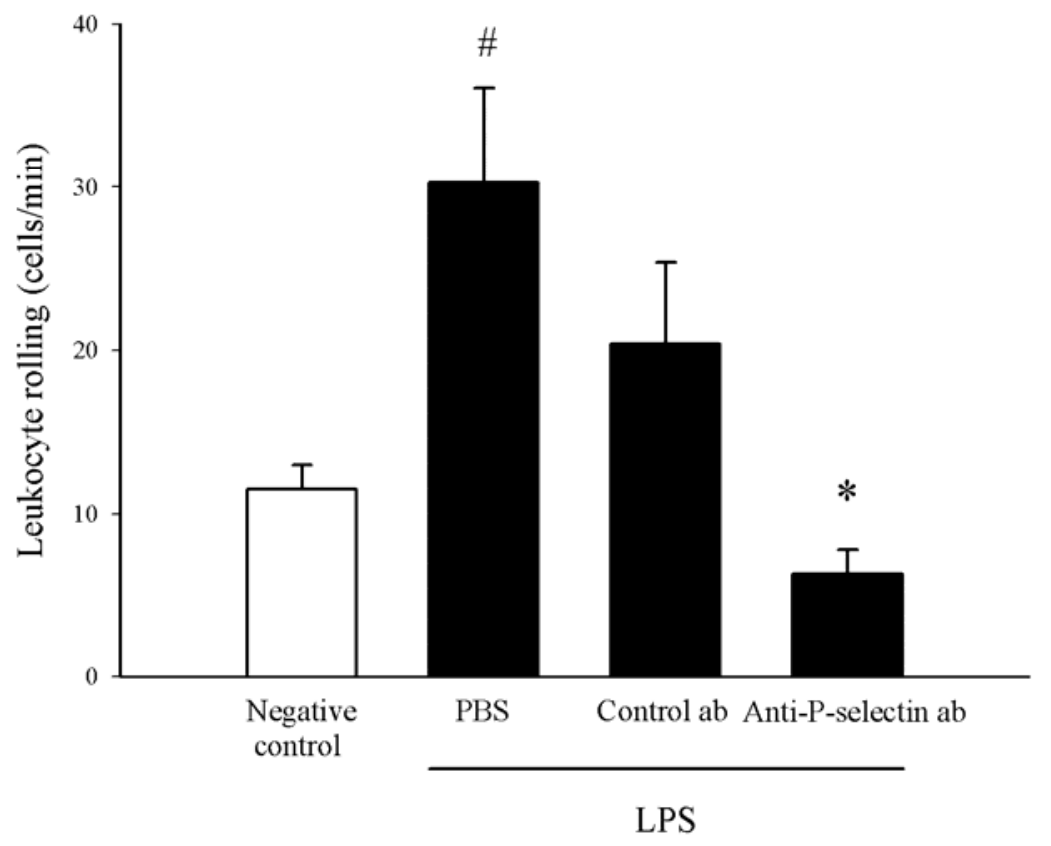




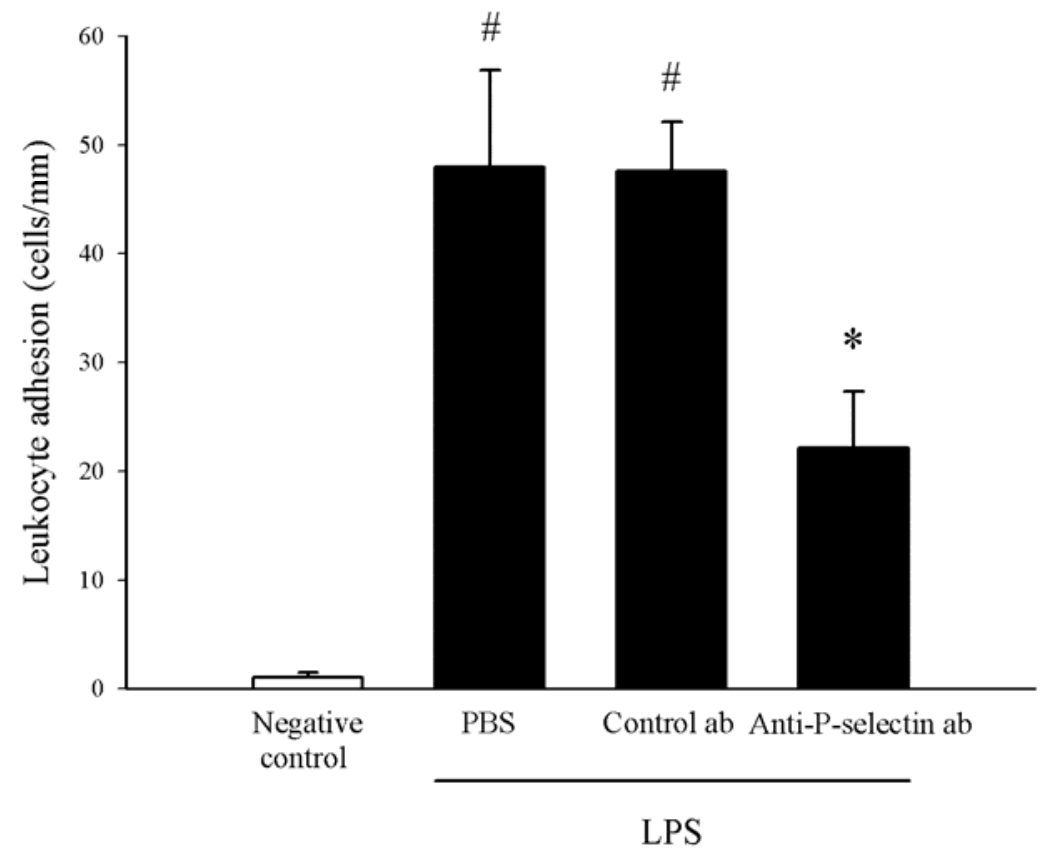




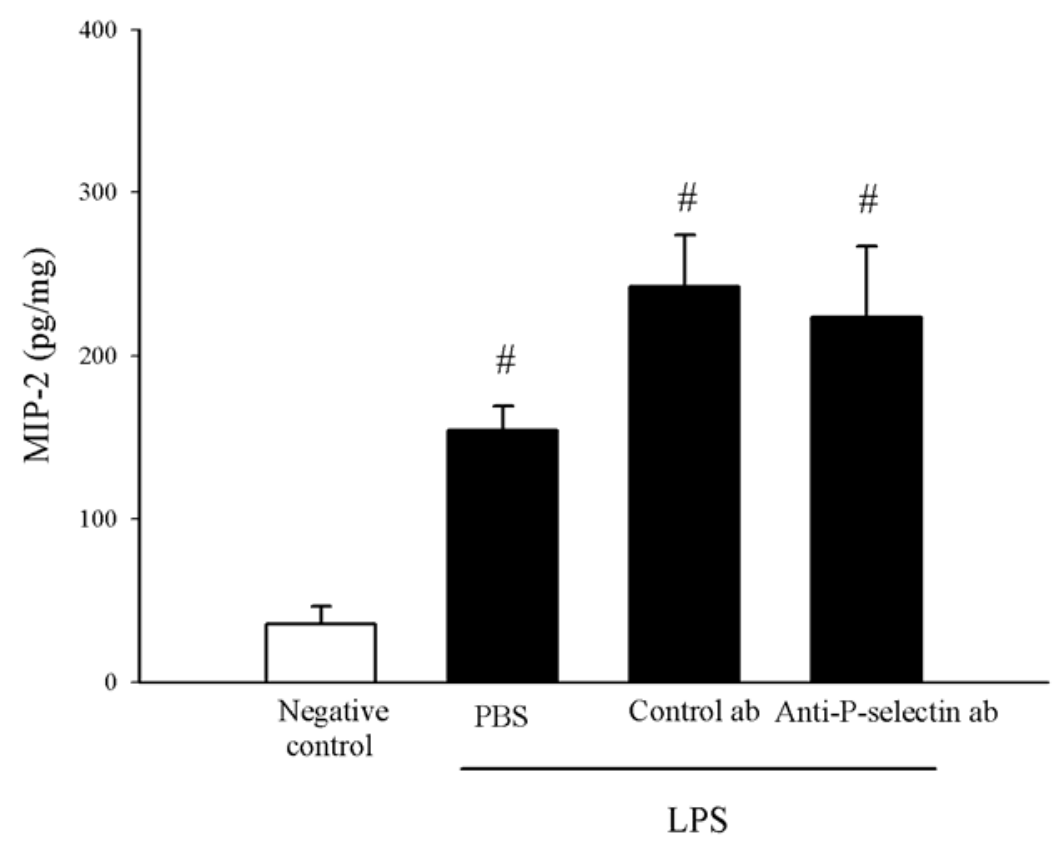




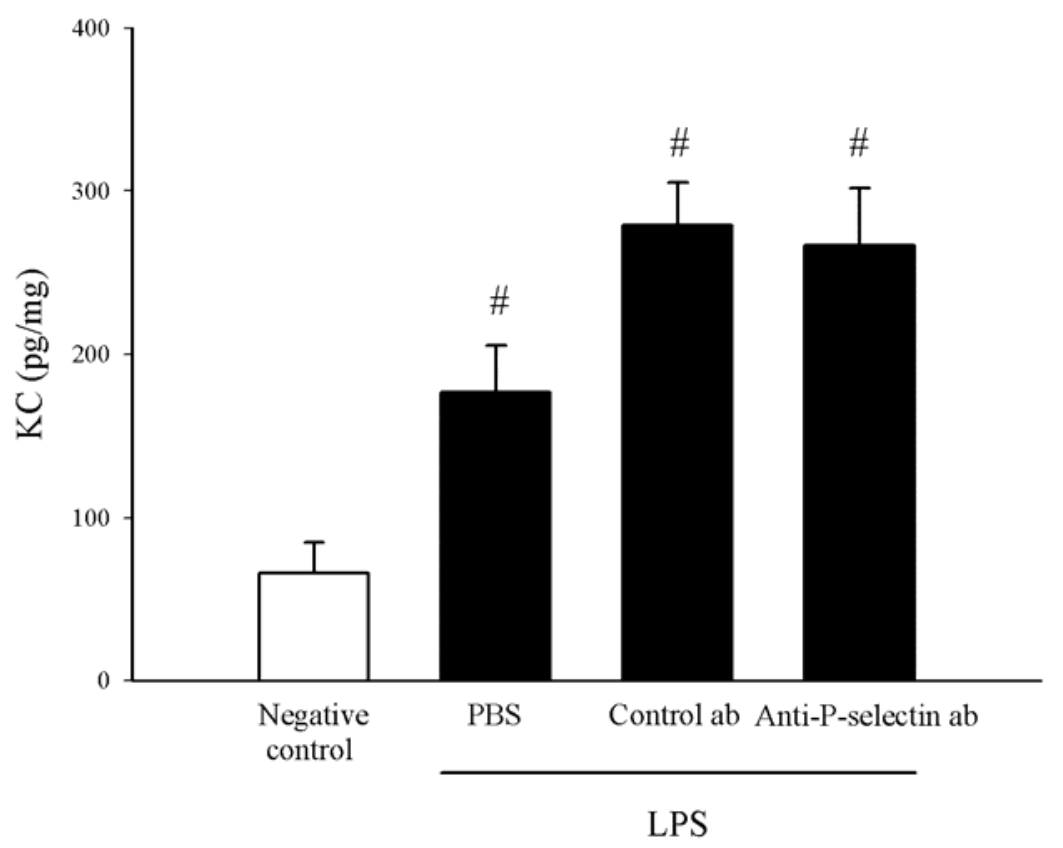

